\title{
EXPANSION OF ANALYTIC FUNCTIONS IN EXPONENTIAL POLYNOMIALS
}

\author{
J. W. LAYMAN
}

The expansion of analytic functions in interpolation series by the method of kernel expansion has been treated in [3], [4] and the detailed application of the method to expansions in the classical polynomials has culminated in the well-known monograph of Boas and Buck [2]. In the present paper we use the kernel expansion technique to obtain a new expansion in which the terms are not polynomials in the usual sense of powers but instead are the exponential polynomials

$$
e_{n}(z)=\sum_{k=0}^{n}(-1)^{k}\left(\begin{array}{l}
n \\
k
\end{array}\right)(n+1-k)^{2} .
$$

These exponential polynomials together with the linear functionals

$$
T_{n}(f)=\left(\begin{array}{l}
\Delta \\
n
\end{array}\right) f(0)=\Delta(\Delta-1) \cdots(\Delta-n+1) f(0) / n !
$$

form a biorthogonal system $\left(T_{n}\left(e_{m}\right)=0\right.$ if $\left.n \neq m, T_{n}\left(e_{n}\right)=1\right)$, giving the formal interpolation series

$$
f(z) \sim \sum_{0}^{\infty}\left(\begin{array}{l}
\Delta \\
n
\end{array}\right) f(0) e_{n}(z) .
$$

Our main result will be the establishment of precise conditions under which (1) actually converges to $f(z)$.

The chief analytical device of the method of kernel expansion is the Pólya representation of analytic functions which we may state as follows: If $f(z)=\sum_{0}^{\infty} a_{n} z^{n} / n$ ! is entire and of exponential type, then

$$
f(z)=(2 \pi i)^{-1} \int_{\Gamma} e^{z w} F(w) d w,
$$

where $F(w)=\sum_{0}^{\infty} a_{n} / w^{n+1}$ and $\Gamma$ encircles the set $D(f)$ consisting of the singular points of $F(w)$ and the points exterior to the domain of $F$. It is well known that $D(f)$ is related to the growth of $f(z)$. (See [1, Chapter 5].)

The representation (2) may also be used to represent many of the most-used linear functionals of analysis in the form

Received by the editors December 6, 1968. 


$$
T_{n}(f)=(2 \pi i)^{-1} \int_{\Gamma} g_{n}(w) F(w) d w
$$

and several instances will be seen below. The function $g_{n}(w)$ is called the generator of $T_{n}$.

Now if the kernel expansion

$$
e^{z w}=\sum_{0}^{\infty} u_{n}(z) g_{n}(w)
$$

holds uniformly for all $w$ on a simple contour $\Gamma$ which encircles $D(f)$ and for all $z$, we may integrate termwise in (2) and obtain $f(z)=\sum_{0}^{\infty} T_{n}(f) u_{n}(z)$, for all $z$.

The method just outlined has been applied by Buck [4] to the classical Newton series. In that case one has the orthonormal system

$$
u_{n}(z)=\left(\begin{array}{c}
z-1 \\
n
\end{array}\right), \quad T_{n}(f)=\Delta^{n} f(1),
$$

yielding the formal expansion

$$
f(z) \sim \sum_{0}^{\infty} \Delta^{n} f(1)\left(\begin{array}{c}
z-1 \\
n
\end{array}\right) .
$$

The representation (3) for $T_{n}$ now can be seen to require $g_{n}(w)=e^{w}\left(e^{w}-1\right)^{n}$. The kernel expansion (4) takes the form

$$
e^{z w}=e^{w}\left[1+\left(e^{w}-1\right)\right]^{z-1}=e^{w} \sum_{0}^{\infty}\left(\begin{array}{c}
z-1 \\
n
\end{array}\right)\left(e^{w}-1\right)^{n}
$$

valid in the region defined by $\left|e^{w}-1\right|<1$. Termwise integration yields the following result. If $f(z)$ is an entire function of exponential type such that $D(f)$ is contained in $\left|e^{w}-1\right|<1$, then $f(z)$ admits the convergent Newton series expansion (5) for all $z$.

The result just given is already stronger than a classical result in Whittaker [8] and still stronger forms are given in [2]. However no result yet obtained by kernel expansion is as strong as that given in Nörlund [7], which we require below and state herewith in somewhat less generality than given in Nörlund.

TheOREM (NöRLUND). If $f(z)$ is analytic and holomorphic in the half-plane $R(z) \geqq \alpha$ and satisfies in that half-plane the inequality $\left|f\left(\alpha+r e^{i \theta}\right)\right|<e^{r \log 2}(1+r)^{\beta+\epsilon(r)},-\pi / 2 \leqq \theta \leqq \pi / 2$, where $\epsilon(r) \rightarrow 0$ as $r \rightarrow \infty$, then $f(z)$ admits the convergent expansion (5) for all $z$ satisfying $R(z)>\max \left(\alpha, \beta+\frac{1}{2}\right)$. Furthermore the convergence is uniform in any bounded subset of the indicated right half-plane of convergence. 
The function $f(z)=z^{\gamma}, \gamma$ any complex number, can be shown (see [5]) to satisfy the conditions required in the previous theorem with $\alpha \geqq \delta>0, \beta=-\frac{1}{2}$. Thus we have

$$
z^{\gamma}=\left.\sum_{0}^{\infty} \Delta^{n} z^{\gamma}\right|_{z=1}\left(\begin{array}{c}
z-1 \\
n
\end{array}\right),
$$

uniformly in any bounded region of the half-plane $R(z) \geqq \epsilon>0$, for arbitrary $\gamma$. It is understood that the branch of $z^{\gamma}$ be chosen consistently throughout (6) so for definiteness we choose the branch satisfying $-\pi / 2<\arg z<\pi / 2$.

An expansion of the form (4) is obtained if we set $z=e^{w}, \gamma=z$ in (6). We have

$$
e^{z w}=\left.\sum_{0}^{\infty} \Delta^{n} x^{z}\right|_{x=1}\left(\begin{array}{c}
e^{w}-1 \\
n
\end{array}\right) .
$$

Uniform convergence is obtained in any bounded region in the strip $|I(w)| \leqq \delta<\pi / 2$. This choice of strip is also consistent with the branch specification above.

The generators $g_{n}(w)$ are now

$$
\left(\begin{array}{c}
e^{w}-1 \\
n
\end{array}\right)
$$

and by virtue of the representation (3) the functionals are found to be

$$
T_{n}(f)=\left(\begin{array}{l}
\Delta \\
n
\end{array}\right) f(0) .
$$

We integrate termwise around a simple contour enclosing $D(f)$ and contained in $|I(w)| \leqq \delta<\pi / 2$. Using the fact that $D(f)$ is closed and bounded for any entire function of exponential type, we obtain our main result, as follows:

THEOREM. Any entire function of exponential type such that $D(f)$ lies in the strip $|I(w)|<\pi / 2$ admits the convergent exponential interpolation series expansion

$$
f(z)=\sum_{0}^{\infty}\left(\begin{array}{l}
\Delta \\
n
\end{array}\right) f(0) e_{n}(z),
$$

for all $z$, where $e_{n}(z)$ is the exponential polynomial

$$
e_{n}(z)=\left.\Delta^{n} x^{z}\right|_{x=1}=\sum_{k=0}^{n}(-1)^{k}\left(\begin{array}{l}
n \\
k
\end{array}\right)(n+1-k)^{z} .
$$


REMARK. It has been pointed out by the referee that Macintyre [6] has given a refinement of the theorem of Nörlund stated above, establishing convergence in $R(z)>\max (\alpha, \beta)$.

\section{BIBLIOGRAPHY}

1. R. P. Boas, Jr., Entire functions, Academic Press, New York, 1954.

2. R. P. Boas, Jr. and R. C. Buck, Polynomial expansions of analytic functions, Springer-Verlag, Berlin, 1964.

3. R. C. Buck, Interpolation and uniqueness of entire functions, Proc. Nat. Acad. Sci. U.S.A. 33 (1947), 288-292.

4. - Interpolation series, Trans. Amer. Math. Soc. 64 (1948), 283-298.

5. W. E. Howell, M.S. Thesis, Virginia Polytechnic Institute, Blacksburg, Va., 1968.

6. S. Macintyre, Transform theory and Newton's interpolation series, Proc. London Math. Soc. (3) 4 (1954), 385-401.

7. N. E. Nörlund, Lȩ̧ons sur les séries d'interpolation, Chapter V, Gauthier-Villars, Paris, 1926.

8. J. W. Whittaker, Interpolatory function theory, Cambridge Univ. Press, London, 1935.

Virginia Polytechnic Institute 